\title{
CORRECTION
}

\section{Correction: Distinguishing swept-source optical coherence tomography findings in active toxoplasmic retinochoroiditis}

Imen Ksiaa (D), Sana Khochtali, Mossaab Mefteh, Manel Ben Fredj, Hajer Ben Amor, Nesrine Abroug and Moncef Khairallah

(c) The Author(s), under exclusive licence to The Royal College of Ophthalmologists 2021

Eye (2021) 35:2911; https://doi.org/10.1038/s41433-021-01692-x

Correction to: Eye

https://doi.org/10.1038/s41433-021-01491-4
Due to a processing error the spelling of given name by Manel Ben Fredj was incorrect.

The original article has been corrected. 